\title{
Multiparameter Operator Systems with Three Parameters
}

\author{
Rakhshanda Dzhabarzadeh $^{1}$, Kamilla Alimardanova ${ }^{2}$ \\ ${ }^{1}$ Department of functional analysis, Institute of Mathematics and Mechanics of NAS of Azerbaijan, Baku \\ ${ }^{2}$ Department of functional analysis of mathematics and Mechanics of NAS of Azerbaijan, Baku
}

Email address:

rakhshanda.dzhabarzade@rambler.ru (R. Dzhabarzadeh), a.kamilla@live.com (K. Alimardanova)

\section{To cite this article:}

Rakhshanda Dzhabarzadeh, Kamilla Alimardanova. Multiparameter Operator Systems with Three Parameters. Pure and Applied Mathematics Journal. Special Issue: Spectral Theory of Multiparameter Operator Pencils and Its Applications. Vol. 4, No. 4-1, 2015, pp. 5-10. doi: 10.11648/j.pamj.s.2015040401.12

\begin{abstract}
For the multiparameter system of operators in three parameters the conditions of the existence of multiple basis of eigen and associated vectors in finite dimensional space is proved. The proof of this fact uses essentially the notion of the Resultant of two operator pencils, acting in, generally speaking, in different Hilbert spaces and the criterion of existence of common eigenvalues of several operator pencils, acting in Hilbert spaces.
\end{abstract}

Keywords: Eigen and Associated Vectors, Finite Dimensional Space, Multiparameter System of Operators, Nonlinear Algebraic System of Equations, Resultant-Operator of Two Pencils

\section{Introduction}

The founder of researches of spectral problems of the multiparameter selfadjoint systems was F.V. Atkinson [1]. Studied the outcomes which are available for multiparameter symmetrical differential systems, Atkinson has constructed the spectral theory of multiparameter systems in finite dimensional spaces. Further, by means of passage to the limit Atkinson has generalized the received outcomes on a case of multiparameter systems with the selfadjoint compact operators in infinite-dimensional Hilbert spaces. In the further, a design introduced by Atkinson for study of multiparameter systems in finite-dimensional spaces, it has appeared possible to build and in infinite-dimensional spaces that has allowed to construct the spectral theory of multiparameter systems in Hilbert spaces [2],[3], etc.

But, unfortunately, the technique of research in these works demands all operators in the system to be selfadjoint.
For non- selfadjoint multiparameter systems the investigated technique does not allow to solve the simplest problems of the spectral theory.

The paper is devoted to the study of nonlinear multiparameter system of operators in finite dimensional Hilbert spaces. Previously, for nonlinear algebraic of system of equations was built analog of determinant of Cramer and it is given a necessary and sufficient condition for the existence of solutions of nonlinear algebraic systems with a complex dependence on the variables [4]. For two-parameter systems in the abstract case, it is obtained the results to determine the conditions for finding the number of solutions [6].

In this paper describes the method of determining the conditions on the coefficients of the multiparameter system to establish the existence of its eigenvalues.

Consider a multiparameter system of operators in three parameter of the form.

$$
\begin{aligned}
& A(\lambda, \mu, \xi) x=\left(A_{0}+\lambda A_{1}+\ldots+\lambda^{m_{1}} A_{m_{1}}+\mu A_{m_{1}+1}+\ldots+\mu^{n_{1}} A_{m_{1}+n_{1}}+\xi A_{m_{1}+n_{1}+1}\right) x=0 \\
& B(\lambda, \mu, \xi) y=\left(B_{0}+\lambda B_{1}+\ldots+\lambda^{m_{1}} B_{m_{2}}+\mu B_{m_{2}+1}+\ldots+\mu^{n_{2}} B_{m_{2}+n_{2}}+\xi B_{m_{2}+n_{2}+1}+\ldots+\xi^{r_{2}} B_{m_{2}+n_{2}+r_{2}}\right) y=0 \\
& C(\lambda, \mu, \xi) z=\left(C_{0}+\lambda C_{1}+\ldots+\lambda^{m_{3}} C_{m_{3}}+\mu C_{m_{3}+1}+\ldots+\mu^{n_{3}} C_{m_{3}+n_{3}}+\xi C_{m_{3}+n_{3}+1}+\ldots+\xi^{r_{3}} C_{m_{3}+n_{3}+r_{3}}\right) z=0
\end{aligned}
$$

when operators $A_{i}, i=1,2, . ., m_{1}+n_{1}+1 \quad$ (correspondingly,

$B_{i}, i=1,2, . ., m_{2}+n_{2}+r_{2}$, and $\left.C_{i}, i=1,2, . ., m_{3}+n_{3}+r_{3}\right)$ act in Hilbert space $H_{i}$ (correspondingly, $H_{2}$ and $H_{3}$ ).

\section{Preliminary definitions and Remarks.}

Let's reduce a series of known positions from the spectral 
theory of multiparameter systems

Definition1. [1,2,3]. An operator $A_{k}^{+}=A_{k} \otimes E_{2} \otimes E_{3}$ (accordingly, $B_{k}^{+}=E_{1} \otimes B_{k} \otimes E_{3}, C_{k}^{+}=E_{1} \otimes E_{2} \otimes C_{k}$ ), where $E_{1}$ (accordingly, $E_{2}$ and $E_{3}$ ) is identical operators in $H_{1}$ (accordingly, $\mathrm{H}_{2}$ and $\mathrm{H}_{3}$ ), is called the operator, induced in $H=H_{1} \otimes H_{2} \otimes H_{3}$ by the operators $A_{k}$ (accordingly, $B_{k}$ and $C_{k}$ ).

Definition 2. [1], [2], [3] $(\lambda, \mu, \xi) \in C^{3}$ is an eigen value of the system(1) if there are such nonzero elements $x \in H_{1}, y \in H_{2}, z \in H_{3}$ that equations (1) and also the equations

$$
\begin{aligned}
& A(\lambda, \mu, \xi)^{+} x \otimes y \otimes z=0 \\
& B(\lambda, \mu, \xi)^{+} x \otimes y \otimes z=0 \\
& C(\lambda, \mu, \xi)^{+} x \otimes y \otimes z=0
\end{aligned}
$$

are satisfied, then the decomposable tensor $x \otimes y \otimes z$ is called the eigenvector of the multiparameter system (1).

Definition 3. [9],[10] A tensor $z_{m_{1}, m_{2}, m_{3}}$ is called the $\left(m_{1}, m_{2}, m_{3}\right)$-associated vector to an eigenvector $z_{0,0,0}=x \otimes y \otimes z$ if there is the set of elements $\left(z_{i, j, k}\right)$ such that the following conditions (2) are satisfied

$$
\operatorname{Re} s(A(\lambda), B(\lambda))=\left(\begin{array}{cccccc}
A_{0} \otimes E_{2} & A_{1} \otimes E_{2} & \ldots & A_{n} \otimes E_{2} & \ldots & 0 \\
. & . & \ldots & . & \ldots & . \\
0 & 0 & \ldots A_{0} \otimes E_{2} & A_{1} \otimes E_{2} & \ldots & A_{n} \otimes E_{2} \\
E_{1} \otimes B_{0} & E_{1} \otimes B_{1} & \ldots & E_{1} \otimes B_{m} & . . & 0 \\
. & . & \ldots & . & \ldots & . \\
. & . & \ldots E_{1} \otimes B_{0} & E_{1} \otimes B_{1} & \ldots & E_{1} \otimes B_{m}
\end{array}\right)
$$

In a matrix $\operatorname{Re}(A(\lambda), B(\lambda))$ the number of rows with operators $A_{i}$ is equal to leading degree of parameter $\lambda$ in the operator pencil $B(\lambda)$, that is $m$; the number of rows in matrix $\operatorname{Re} s(A(\lambda), B(\lambda))$ with operators $B$ coincides with the leading degree of parameter $\lambda$ in the operator pencil $A(\lambda)$, that is $n$. One major application of the matrix theory is calculation of determinants. It turns out that a mapping is invertible if and only if the determinant of this matrix is not zero.

This definition is the generalization of the notion of the definition of resultant for two polynomials

$$
\begin{aligned}
& f(x), g(x) ; f(x)=a_{n} x^{n}+a_{n-1} x^{n-1}+\ldots+a_{0}, \quad a_{n} \neq 0 ; \\
& g(x)=b_{m} x^{m}+b_{m-1} x^{m-1}+\ldots+b_{0}, b_{m} \neq 0 ;
\end{aligned}
$$

Resultant of these polynomials is the operator acting in the space $R^{n+m}$ or $C^{n+m}$ (probably, in some expansion of a field).

$$
\begin{aligned}
& \sum_{0 \leq r_{i} \leq k_{i}} \frac{1}{r_{1} ! r_{2} ! r_{3} !} \frac{\partial^{r_{1}+r_{2}+r_{3}} A^{+}(\lambda, \mu, \xi)}{\partial^{r_{1}} \lambda \partial^{r_{2}} \mu \partial^{r_{3}} \xi} z_{k_{1}-r_{1}, k_{2}-r_{2}, k_{3}-r_{3}}=0 \\
& \sum_{0 \leq r_{i} \leq k_{i}} \frac{1}{r_{1} ! r_{2} ! r_{3} !} \frac{\partial^{r_{1}+r_{2}+r_{3}} B^{+}(\lambda, \mu, \xi)}{\partial^{r_{1}} \lambda \partial^{r_{2}} \mu \partial^{r_{3}} \xi} z_{k_{1}-r_{1}, k_{2}-r_{2}, k_{3}-r_{3}}=0 \\
& \sum_{0 \leq r_{i} \leq k_{i}} \frac{1}{r_{1} ! r_{2} ! r_{3} !} \frac{\partial^{r_{1}+r_{2}+r_{3}} C^{+}(\lambda, \mu, \xi)}{\partial^{r_{1}} \lambda \partial^{r_{2}} \mu \partial^{r_{3}} \xi} z_{k_{1}-r_{1}, k_{2}-r_{2}, k_{3}-r_{3}}=0 \\
& k_{s} \leq m_{s} ; i=1,2,3 ; s=1,2,3 .
\end{aligned}
$$

$\left(k_{1}, k_{2}, k_{3}\right)$ is arrangement from set of the whole nonnegative numbers on 3 with possible recurring and zero.

Remark 1. We gave here the definition of the associated vector for the case when the number of parameters in the system is equal to 3 (in [10] the definition is given for the common case)

Definition 4. [11],[13]

Let

$$
\begin{aligned}
& A(\lambda)=A_{0}+\lambda A_{1}+\lambda^{2} A_{2}+\ldots+\lambda^{n} A_{n} \\
& B(\lambda)=B_{0}+\lambda B_{1}+\lambda^{2} B_{2}+\ldots+\lambda^{m} B_{m}
\end{aligned}
$$

be two operator pencils depending on the same parameter $\lambda$ and acting, generally speaking, in different Hilbert spaces $H_{1}, H_{2}$, correspondingly. Resultant of two operator pencils $A(\lambda)$ and $B(\lambda)$ is the operator, presented by the determinant (4) and acting in the space $\left(H_{1} \otimes H_{2}\right)^{n+m}$ - direct sum of $n+m$ copies of tensor product $H_{1} \otimes H_{2}$ of spaces

$$
\operatorname{Re} s(f, g)=\left(\begin{array}{cccccccccc}
a_{n} & a_{n-1} & \ldots & a_{1} & a_{0} & 0 & \ldots & \ldots & 0 & 0 \\
0 & a_{n} & \ldots & a_{2} & a_{1} & a_{0} & \ldots & \ldots & 0 & 0 \\
\cdot & . & \ldots & . & . & . & \ldots & \ldots & . & . \\
. & . & \ldots & . & . & . & \ldots & \ldots & . & . \\
0 & 0 & \ldots & 0 & 0 & a_{n} & \ldots & \ldots & a_{1} & a_{0} \\
b_{m} & b_{m-1} & \ldots & b_{3} & b_{2} & b_{1} & b_{0} & \ldots & 0 & 0 \\
0 & b_{m} & \ldots & b_{4} & b_{3} & b_{2} & b_{1} & b_{0} \ldots & 0 & 0 \\
& . & \ldots & . & . & . & . & \ldots & . & . \\
& . & \ldots & . & . & . & . & \ldots & . & . \\
& 0 & 0 & 0 & 0 & b_{m} & b_{m-1} & \ldots & b_{1} & b_{0}
\end{array}\right)
$$

In a matrix $\operatorname{Re} s(f, g)$ the number of rows with coefficients $a_{i}$ to equally leading degree of unknown $x$ of a polynomial $g(x)$, that is $m$, the number of rows of a matrices with numbers $b_{i}$ coincides with the leading degree 
of unknown $x$ of a polynomial $f(x)$, that is $n$. Continuant of this Resultant is a polynomial from coefficients and equal to zero then and only then when polynomials also have a common roots (probably, in some expansion of a field).

The study of multiparameter system (1) of equations is spent with help of following result from [5]:

\section{On common Eigenvalues of Several Operator Bundles}

Let the $n$ bundles depending on the same parameter $\lambda$

$$
\left\{B_{i}(\lambda)=B_{0, i}+\lambda B_{1, i}+\ldots+\lambda^{k_{i}} B_{k_{i}, i}, \quad i=1,2, \ldots, n\right.
$$

$B_{i}(\lambda)$ - operator bundles acting in Hilbert space $H_{i}$ correspondingly. Suppose that $k_{1} \geq k_{2} \geq \ldots \geq k_{n}$. In the space $H^{k_{1}+k_{2}}$ (the direct sum of $k_{1}+k_{2}$ tensor product $H=H_{1} \otimes \ldots \otimes H_{n}$ of spaces $\left.H_{1}, H_{2}, \ldots, H_{n}\right)$ are introduced the operators $R_{i}(i=1, \ldots, n-1)$ with the help of operational matrices (4).

Let $B_{i}(\lambda)$ be the operational bundles acting in a finite dimensional Hilbert space $H_{i}$, correspondingly.

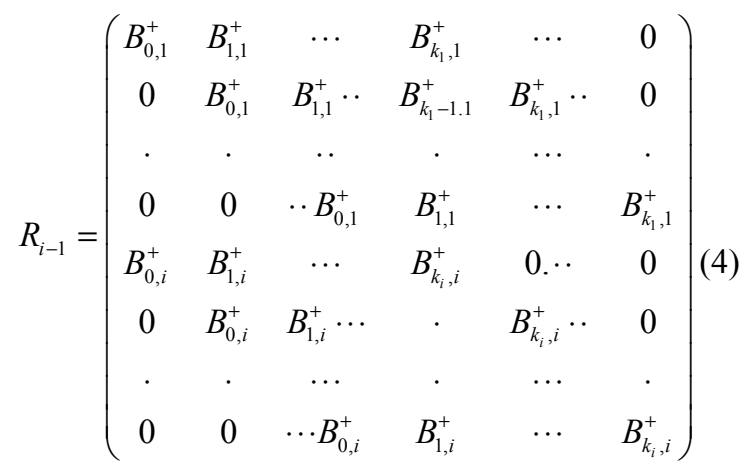

$i=2,3, \ldots, n$

The number of rows with operators $B_{s, 1}, s=0,1, \ldots, k_{1}$ in the matrix $R_{i-1}$ is equal to $k_{2}$ and the number of rows with operators $B_{s, i}, s=0,1, \ldots, k_{i}$ is equal to $k_{1}$. We designate $\sigma_{p}\left(B_{i}(\lambda)\right)$ the set of eigenvalues of an operator $B_{i}(\lambda)$.From [5] we have the result:

Theorem 1. $\bigcap_{i=1}^{n} \sigma_{p}\left(B_{i}(\lambda)\right) \neq\{\theta\} \quad$ if $\quad$ and $\quad$ only $\quad$ if $\bigcap_{i=1}^{n-1} \operatorname{KerR}_{i} \neq\{\theta\},\left(\operatorname{KerB}_{k_{1}}=\{\theta\}\right)$.

In particular, in [6] the two-parameter system operators

$$
\begin{aligned}
& A(\lambda, \mu) x=\left(A_{0}+\lambda A_{1}+\ldots+\lambda^{m_{1}} A_{m_{1}}+\mu A_{m_{1}+1}+\ldots+\mu^{n_{1}} A_{m_{1}+n_{1}}\right) x=0 \\
& B(\lambda, \mu) y=\left(B_{0}+\lambda B_{1}+\ldots+\lambda^{m_{2}} B_{m_{2}}+\mu B_{m_{2}+1}+\ldots+\mu^{n_{2}} B_{m_{2}+n_{2}}\right) y=0
\end{aligned}
$$

in tensor product $H_{1} \otimes H_{2}$ of finite-dimensional spaces $H_{1}$ and $\mathrm{H}_{2}$ is studied.

Dimension of space $H$ is the product of dimensions of spaces $H_{1}$ and $H_{2}$. In (5) linear operators $A_{i}\left(i=0,1, \ldots, m_{1}+n_{1}\right)$ act in finite-dimensional space $H_{1}$; and linear operators $B_{i}\left(i=0,1, \ldots, m_{2}+n_{2}\right)$ act in finitedimensional space $\mathrm{H}_{2}$.

If $f_{1} \otimes f_{2} \in H_{1} \otimes H_{2}$ and $g_{1} \otimes g_{2} \in H_{1} \otimes H_{2}$ then inner product of these elements in space $H_{1} \otimes H_{2}$ is defined by means of the formulae

$$
\left[f_{1} \otimes f_{2}, g_{1} \otimes g_{2}\right]_{H_{1} \otimes H_{2}}=\left(f_{1}, g_{1}\right)_{H_{1}} \cdot\left(g_{1}, g_{2}\right)_{H_{2}}
$$

This definition of inner product is spread to other elements of tensor product space $H_{1} \otimes H_{2}$ on linearity.

If $H_{1} \otimes H_{2}$ is the Hilbert space then the inner product (6) is spread to other elements of $H_{1} \otimes H_{2}$ on linearity and continuity.

By means of the approach stated in $[5,6]$, we can establish completeness, multiple completeness of system of eigen and associated vectors, a possibility of multiple decompositions on system of eigen and associated vectors of multiparameter system (5).

Theorem 2. [6] Let operators $A_{i}\left(i=0,1, \ldots, m_{1}+n_{1}\right)$ also $B_{i}\left(i=0,1, \ldots, m_{2}+n_{2}\right)$ act in finite-dimensional spaces $H_{1}$ and $H_{2}$, accordingly, and one of three following conditions is fulfilled:

a) $\max \left(m_{1} n_{2}, m_{2} n_{1}\right)=m_{1} n_{2}, \operatorname{Ker}_{m_{1}}=\{\theta\}, \operatorname{Ker} B_{m_{2}+n_{2}}=\{\theta\}$; $A_{m_{1}}, B_{m_{2}+n_{2}}$ are self-adjoint operators everyone in their space

b) $\max \left(m_{1} n_{2}, m_{2} n_{1}\right)=m_{2} n_{1}, \operatorname{Ker} B_{m_{2}}=\{\theta\}, \operatorname{Ker}_{m_{1}+n_{1}}=\{\theta\}$

$A_{m_{1}+n_{1}}, B_{m_{1}}$ - self-adjoint operators, acting in the corresponding spaces

c) $m_{1} n_{2}=m_{2} n_{1}, \operatorname{Ker}\left(A_{m_{1}}^{n_{2}} \otimes B_{m_{1}+n_{1}}^{n_{1}}+(-1)^{n_{1} n_{2}} A_{m_{1}+n_{1}}^{n_{2}} \otimes B_{m_{2}}^{n_{1}}\right)$, $A_{m_{1}}, B_{m_{2}}, A_{m_{1}+n_{1}}, B_{m_{2}+n_{2}}$ are the self-adjoint operators, acting everyone in finite-dimensional space, correspondingly.

Then the $\max \left(m_{1} n_{2}, m_{2} n_{1}\right)$-fold basis of system of eigen and associated vectors of (5) takes place.

At the study of the system (1) we will be used essentially the results of $[5,6]$ :

\section{Three Parameter System of Operators in Finite Dimensional Hilbert Spaces}

In (1) we fix parameters $\lambda, \mu$. Let be $\lambda=\lambda_{0}, \mu=\mu_{0}$. Then the system (1) contains three operator bundles depending on one parameter $\xi$. Using the result of the theorem 2, we build operators $R_{1}$ и $R_{2}$.They are the Resultants of operator bundles $A\left(\lambda_{0}, \mu_{0}, \xi\right)$ and $B\left(\lambda_{0}, \mu_{0}, \xi\right)$, 
and also operator bundles $A\left(\lambda_{0}, \mu_{0}, \xi\right)$ and $C\left(\lambda_{0}, \mu_{0}, \xi\right)$, Introduce the notations: correspondingly.

$$
\begin{aligned}
& \tilde{A}\left(\lambda_{0}, \mu_{0}\right)=A_{0}+\lambda_{0} A_{1}+\ldots+\lambda_{0}^{m_{1}} A_{m_{1}}+\mu_{0} A_{m_{1}+1}+\ldots+\mu_{0}^{n_{1}} A_{m_{1}+n_{1}} \\
& \tilde{B}\left(\lambda_{0}, \mu_{0}\right)=B_{0}+\lambda_{0} B_{1}+\ldots+\lambda_{0}^{m_{2}} B_{m_{2}}+\mu_{0} B_{m_{2}+1}+\ldots+\mu_{0}^{n_{2}} B_{m_{2}+n_{2}} \\
& C\left(\lambda_{0}, \mu_{0}\right)=C_{0}+\lambda_{0} C_{1}+\ldots+\lambda_{0}^{m_{3}} C_{m_{3}}+\mu_{0} C_{m_{3}+1}+\ldots+\mu_{0}^{n_{3}} C_{m_{3}+n_{3}}
\end{aligned}
$$

Let $r_{2} \geq r_{3}$. For the polynomials $A\left(\lambda_{0}, \mu_{0}, \xi\right)$ and $B\left(\lambda_{0}, \mu_{0}, \xi\right)$ Resultant $R_{1}$ has a form

$$
\begin{aligned}
& R_{1}=R\left\{A\left(\lambda_{0}, \mu_{0}, \xi\right), C\left(\lambda_{0}, \mu_{0}, \xi\right)\right\}= \\
& \left(\begin{array}{ccccc}
\widetilde{A}^{+}\left(\lambda_{0}, \mu_{0}\right) & A_{m_{1}+n_{1}+1}^{+} & . & \ldots & . \\
0 & \widetilde{A}^{+}\left(\lambda_{0}, \mu_{0}\right) & A_{m_{1}+n_{1}+1}^{+} & \ldots & 0 \\
\cdot & \cdot & \cdot & \ldots & . \\
\cdot & \cdot & \cdot & . . A_{m_{1}+n_{1}+1}^{+} \cdot & 0 \\
B^{+}\left(\lambda_{0}, \mu_{0}\right) & B_{m_{3}+n_{3}+1}^{+} & B_{m_{3}+n_{3}+2}^{+} & \ldots & B_{m_{3}+n_{3}+r_{3}}^{+}
\end{array}\right)
\end{aligned}
$$

In the matrices of the operator $R_{1}$ the number of rows with $\tilde{A}^{+}\left(\lambda_{0}, \mu_{0}\right), A_{m_{1}+n_{1}+1}^{+}$equal to $r_{2}$ and the number of rows with $B^{+}\left(\lambda_{0}, \mu_{0}\right), \quad B_{m_{2}+n_{2}+1}^{+}, \ldots, B_{m_{2}+n_{2}+r_{2}}^{+}$is equal to 1

By analogy operator $R_{2}$ in the space $\left(H_{1} \otimes H_{2} \otimes H_{3}\right)^{r_{2}+1}$ is determined with the help of matrices

$$
\begin{aligned}
& R_{1}=R\left\{A\left(\lambda_{0}, \mu_{0}, \xi\right), C\left(\lambda_{0}, \mu_{0}, \xi\right)\right\}= \\
& \left(\begin{array}{ccccc}
\widetilde{A}^{+}\left(\lambda_{0}, \mu_{0}\right) & A_{m_{1}+n_{1}+1}^{+} & \cdot & \ldots & \cdot \\
0 & \widetilde{A}^{+}\left(\lambda_{0}, \mu_{0}\right) & A_{m_{1}+n_{1}+1}^{+} & \ldots & 0 \\
\cdot & \cdot & \cdot & \ldots & \cdot \\
\cdot & \cdot & \cdot & . . A_{m_{1}+n_{1}+1}^{+} \cdot & 0 \\
C^{+}\left(\lambda_{0}, \mu_{0}\right) & C_{m_{3}+n_{3}+1}^{+} & C_{m_{3}+n_{3}+2}^{+} & \ldots & C_{m_{3}+n_{3}+r_{3}}^{+}
\end{array}\right)
\end{aligned}
$$

In the matrix of the operator $R_{2}$ the number of rows with $\tilde{A}^{+}\left(\lambda_{0}, \mu_{0}\right), A_{m_{1}+n_{1}+1}^{+}$is equal to $r_{2}$ and the number of rows with $C^{+}\left(\lambda_{0}, \mu_{0}\right), \quad C_{m_{3}+n_{3}+1}^{+}, \ldots, C_{m_{3}+n_{3}+r_{3}}^{+}$is equal to 1

Operators $R_{1}$ and $R_{2}$ act in the same space $\left(H_{1} \otimes H_{2} \otimes H_{3}\right)^{r_{2}+1}$ - direct sum of the $r_{2}+1$ copies of tensor product space $H_{1} \otimes H_{2} \otimes H_{3}$.

Continuants of Resultants $R_{1}$ and $R_{2}$ are equal to zero if and only if matrices of operators $R_{1}$ and $R_{2}$ have nonzero kernels.

So at the decompositions of continuants of Resultants $R_{1}$ and $R_{2}$ we obtain very bulky forms, in obtained equations we will operate with the leading degrees of parameters in $R_{1}$ and $R_{2}$. Further we will work only with the members having the leading degrees. We can write the decompositions of continuants of Resultants $R_{1}$ and $R_{2}$ in the forms, correspondingly:

$$
\begin{aligned}
& \ldots+\lambda_{0}^{m_{1} r_{2}}\left(A_{m_{1}}^{r_{2}}\right)^{+} B_{m_{2}+n_{2}+r_{2}}^{+}+\ldots+\mu_{0}^{n_{1} r_{1}}\left(A_{m_{1}+n_{1}}^{r_{2}}\right)^{+} B_{m_{2}+n_{2}+r_{2}}^{+}-\ldots-\lambda_{0}^{m_{2}}\left(A_{m_{1}+n_{1}+1}^{r_{2}}\right)^{+} B_{m_{2}}^{+}-\ldots-\mu_{0}^{r_{2}}\left(A_{m_{1}+n_{1}+1}^{r_{2}}\right)^{+} B_{m_{2}+n_{2}}^{+} \\
& \ldots+\lambda_{0}^{m_{1} r_{3}}\left(A_{m_{1}}^{r_{2}}\right)^{+} C_{m_{3}+n_{3}+r_{3}}^{+}+\ldots+\mu_{0}^{n_{1} r_{2}}\left(A_{m_{1}+n_{1}}^{r_{2}}\right)^{+} C_{m_{3}+n_{3}+r_{3}}^{+}-\ldots-\lambda_{0}^{m_{2}}\left(A_{m_{1}+n_{1}+1}^{r_{2}}\right)^{+} C_{m_{3}}^{+}-\ldots-\mu_{0}^{r_{2}}\left(A_{m_{1}+n_{1}+1}^{r_{2}}\right)^{+} C_{m_{3}+n_{3}}^{+}
\end{aligned}
$$

Expression (9) is the decomposition of the continuant of the Resultant of the operator bundles $A\left(\lambda_{0}, \mu_{0}, \xi\right)$ and $B\left(\lambda_{0}, \mu_{0}, \xi\right)$ from (1), when $\lambda=\lambda_{0}$ and $\mu=\mu_{0}$. Expression (10) is the decomposition of the continuant of the Resultant of operator bundles $A\left(\lambda_{0}, \mu_{0}, \xi\right)$ and $C\left(\lambda_{0}, \mu_{0}, \xi\right)$. Let the couple $\left(\lambda_{1}, \mu_{1}\right)$ is the eigen value of the (9), then from the definition of Resultant follows that at these meanings of parameters $\lambda=\lambda_{1}$ and $\mu=\mu_{1}$ we have that operator pencils $A\left(\lambda_{1}, \mu_{1}, \xi\right)$ and $B\left(\lambda_{1}, \mu_{1}, \xi\right)$ have a common point of spectra $\xi_{1}\left(\lambda_{1}, \mu_{1}\right)$. By analogy if the couple $\left(\lambda_{2}, \mu_{2}\right)$ is the eigen value of the (10), then from the definition of Resultant follows that at these meanings of parameters $\lambda=\lambda_{2}$ and $\mu=\mu_{2}$ we have that operator pencils $A\left(\lambda_{2}, \mu_{2}, \xi\right)$ and 
$C\left(\lambda_{2}, \mu_{2}, \xi\right)$ have a common point $\xi_{2}\left(\lambda_{2}, \mu_{2}\right)$ of spectra of Now we consider the system of equations these operator bundles.

$$
\begin{aligned}
& \left(\ldots+\lambda_{0}^{m_{1} r_{2}}\left(A_{m_{1}}^{r_{2}}\right)^{+} B_{m_{2}+n_{2}+r_{2}}^{+}+\ldots+\mu_{0}^{n_{1} r_{2}}\left(A_{m_{1}+n_{1}}^{r_{2}}\right)^{+} B_{m_{2}+n_{2}+r_{2}}^{+}-\ldots-\lambda_{0}^{m_{2}}\left(A_{m_{1}+n_{1}+1}^{r_{2}}\right)^{+} B_{m_{2}}^{+}-\ldots-\mu_{0}^{r_{2}}\left(A_{m_{1}+n_{1}+1}^{r_{2}}\right)^{+} B_{m_{2}+n_{2}}^{+}\right) \tilde{u}=0 \\
& \left(\ldots+\lambda_{0}^{m_{1} r_{3}}\left(A_{m_{1}}^{r_{2}}\right)^{+} C_{m_{3}+n_{3}+r_{3}}^{+}+\ldots+\mu_{0}^{n_{1} r_{2}}\left(A_{m_{1}+n_{1}}^{r_{2}}\right)^{+} C_{m_{3}+n_{3}+r_{3}}^{+}-\ldots-\lambda_{0}^{m_{3}}\left(A_{m_{1}+n_{1}+1}^{r_{2}}\right)^{+} C_{m_{3}}^{+}-\ldots-\mu_{0}^{r_{3}}\left(A_{m_{1}+n_{1}+1}^{r_{2}}\right)^{+} C_{m_{3}+n_{3}}^{+}\right) \tilde{u}=0
\end{aligned}
$$

If the $(\lambda, \mu)$ is the eigenvalue of the system (11) and $\tilde{u}$ is the corresponding eigen vector of (11) then $\operatorname{KerR}_{1} \cap R_{2} \neq\{\theta\}$. So the [8], [9] are decompositions of Resultants $R_{1}$ and $R_{2}$, correspondingly, and (11) is satisfied then the element $\widetilde{u}$ is the first component of the element of $\operatorname{Ker} R_{1} \cap R_{2}$ and also the eigen vector of the system (1) or its associated vector in definition of which no the differential on $\lambda$ and $\mu$.All the associated vectors of the system (11) are also the associated vectors of the system (1).

Operator pencils (9) and (10) form the nonlinear two parameter system of the kind of the system (5). (10)

We apply the results of the theorem 2 to the system (9),

Theorem 3. Let operators $A_{i}\left(i=0,1, \ldots, m_{1}+n_{1}+1\right)$, $B_{i}\left(i=0,1, \ldots, m_{2}+n_{2}+r_{2}\right)$ and also $C_{i}\left(i=0,1, \ldots, m_{3}+n_{3}+r_{3}\right)$ act in finite-dimensional spaces $H_{1}, H_{2}$ and $H_{3}$, accordingly, and one of three following conditions is fulfilled: a) $\max \left(m_{1} r_{2} r_{3}, m_{2} r_{3}, m_{3} n_{1} r_{2}\right)=m_{1} r_{2} r_{3}$ $\operatorname{Ker}_{m_{1}}=\{\theta\}, \operatorname{Ker}_{m_{1}+n}=\{\vartheta\}, \operatorname{Ker} B_{m_{2}+n_{2}+r_{2}}=\{\theta\}, \operatorname{Ker} C_{m_{3}+n_{3}}=\{\vartheta\} \quad ;$ $A_{m_{1}}^{r_{2}} A_{m_{1}+n_{1}+1}^{r_{2}} \otimes B_{m_{2}+n_{2}} \otimes C_{m_{3}+n_{3}}$ are self-adjoint operator in the space $H_{1} \otimes H_{2} \otimes H_{3}$

b) $\max \left(m_{1} r_{2} r_{3}, m_{2} r_{3}, m_{3} n_{1} r_{2}\right)=m_{2} r_{3}$

$\operatorname{Ker} B_{m_{2}}=\{\theta\}, \operatorname{Ker} A_{m_{1}+n_{1}+1}=\{\theta\}, \operatorname{Ker} C_{m_{3}+n_{3}}=\{\vartheta\}$

$A_{m_{1}+n_{1}+1}^{2 r_{2}} \otimes B_{m_{2}} \otimes C_{m_{3}+n_{3}}$, are the self-adjoint operator, acting in finite-dimensional space $H_{1} \otimes H_{2} \otimes H_{3}$, correspondingly. $\max \left(m_{1} r_{2} r_{3}, m_{2} r_{3}, m_{3} n_{1} r_{2}\right)=m_{3} n_{1} r_{2}$ $\operatorname{Ker} B_{m_{2}+n_{2}+r_{2}}=\{\theta\}, \operatorname{Ker}_{m_{1}+n_{1}+1}=\{\theta\}, \operatorname{Ker} C_{m_{3}}=\{\vartheta\}, \operatorname{Ker} A_{m_{1}+n_{1}}=\{\vartheta\}$ $A_{m_{1}+n_{1}}^{r_{2}} A_{m_{1}+n_{1}=1}^{r_{2}} \otimes B_{m_{2}+n_{2}+r_{2}} \otimes C_{m_{3}}$ is selfadjoint operator in the space $H_{1} \otimes H_{2} \otimes H_{3}$, Then the eigen and associated vectors of the three parameter system of operators in finite dimensional space form the $\max \left(m_{1} r_{2} r_{3}, m_{2} r_{3}, m_{3} n_{1} r_{2}\right)=m_{3} n_{1} r_{2} \quad$ multiple basis in $H_{1} \otimes H_{2} \otimes H_{3}$.

Proof of the theorem 3 uses the approach applying of the proof of the theorem 2 . We fix one of the parameters of the system (9),(10). For the definite let it is the parameter $\lambda$. Then we have two operator bundles depending on the parameter $\mu$. Construct the resultant of (9) and (10), when parameter $\lambda$ is fixed. It is clear that the decomposition of this resultant is the operator bundle. In fact, the leading degree of the parameter $\lambda$ depends on values of numbers $m_{1}, m_{2}, m_{3}, n_{1}, n_{2}, n_{3}, r_{1}, r_{2}, r_{3}$. Conditions a), b) and c) are only some variants of possibility. If in each of the cases a), b) and c) operator coefficient at the parameter $\lambda$ with the greatest degree $\lambda$ is selfadjoint operator with the zero kernel then the system of eigen and associated vectors of this bundle forms the multiple basis in the tensor product space. The multiple of the system of eigen and associated vectors coincides with the leading degree of the parameter $\lambda$. In works [8] and [9] it is proved that the system of eigen and associated vectors of decomposition of resultant coincides with the system of eigen and associated vectors of two parameter system (9),(10).

So the (9) and (10) are also decompositions of corresponding of resultants, we obtain that the system of eigen and associated vectors of (9) and (10) coincides with the system of eigen and associated vectors of the three parameter system (1).

\section{Conclusion}

In this paper the conditions of existence of multiple basis on eigen and associated vectors of three parameter operator system in finite dimensional spaces are proved.

\section{References}

[1] Atkinson F.V. Multiparameter spectral theory. Bull. Amer. Math. Soc. 1968, 74, 1-27.

[2] Browne P.J. Multiparameter spectral theory. Indiana Univ. Math. J,24, 3, 1974

[3] Sleeman B.D. Multiparameter spectral theory in Hilbert space. Pitnam Press, London, 1978, p.118.

[4] Dzhabarzadeh R. M., Salmanova G. H. About solutions of algebraic systems, Proceeding of IMM of NAS of Azerbaijan, 2010, vol. XXX(XLI), pp.43-48

[5] Dzhabarzadeh R. M. On existence of common eigen value of some operator-bundles, that depends polynomial on parameter. Baku. International Topology conference, 3-9 oct., 1987, Tez. 2, Baku, 1987, p.93.

[6] Dzhabarzadeh R. M. On solutions of nonlinear algebraic systems with two variables. Pure and Applied Mathematics Journal, vol. 2, No. 1, pp. 32-37, 2013

[7] Dzhabarzadeh R M. Nonlinear algebraic equations Lambert Academic Publishing, 2013, p. 101 (in Russian)

[8] Dzhabarzadeh R. M. Spectral theory of two parameter system in finite-dimensional space. Transactions of NAS of Azerbaijan, v. 3-4 1998, p.12-18

[9] Dzhabarzadeh R.M. Spectral theory of multiparameter system of operators in Hilbert space, Transactions of NAS of Azerbaijan, 1-2, 1999, 33-40. 
[10] Dzhabarzadeh R.M. Nonlinear algebraic system with three unknowns variables. International Journal of Research Engineering and Science (IJRES), www.ijres.org, Volume2, Issue 6,14 June, 2014, pp 54-59

[11] Balinskii A.I (Балинский) Generation of notions of Bezutiant and Resultant DAN of Ukr. SSR, ser.ph.-math and tech. of sciences, 1980,2. (in Russian).
[12] Keldish M .V. On completeness of eigen functions of some classes of linear nonselfadjoint operators .Successes of Mathematical Sciences (УМH), 1971, v.27, issue.4, pp..1547,(in Russian)

[13] Khayniq (Хайниг Г). Abstract analog of Resultant for two polynomial bundles Functional analyses and its applications, 1977, 2,no. 3, p.94-95(in Russian) 